\title{
Microstructure and magnetic properties of zinc ferrite thin films produced by pulsed laser deposition*
}

\author{
D RAVINDER \\ Department of Physics, Osmania Universit Hyderabad 500 007, India \\ MS received 4 December 1998
}

\begin{abstract}
Zinc ferrite thin films were deposited from a target of zinc ferrite onto a $\mathrm{MgO}$ substrate using $\mathrm{XeCl}$ excimer laser operating at $308 \mathrm{~nm}$ and frequency of $30 \mathrm{~Hz}$. The crystallographic characterizations of the films were performed using X-ray diffraction (XRD). Microstructure, surface morphology, chemical composition and grain size, as well as surface roughness were obtained from scanning electron microscope (SEM), energy dispersive spectroscopy (EDS) and atomic force microscopy (AFM). The magnetic properties of the thin films were studied in the temperature range 5-300 $\mathrm{K}$ and in fields of up to $5 \mathrm{~T}$ using SQUID magnetometry. Data on temperature and field dependence of magnetization provide a strong evidence for superparamagnetism.
\end{abstract}

Keywords. Pulsed laser deposition; magnetic properties; ferrite thin films.

\section{Introduction}

New communication systems use increasingly higher frequencies and smaller and lighter possible devices. This requires new magnetic devices using thin film inductors or transformers and microwave integrated non-reciprocal circuits with incorporated thin ferrite films. Due to their better high frequency characteristics, the polycrystalline materials are preferred to the monocrystalline ones in such thin film applications.

Pulsed laser deposition (PLD) has now proved to be a very useful technique for obtaining thin films of ferrimagnetic oxides (Welech et al 1995), perovskite oxides (Sunita Gangopadhyay et al 1995) and high temperature superconductors (Jackson and Palmer 1994). Magnetic ferrite thin films having the spinels, hexagonal and garnet structure are very useful for applications, especially in the field of passive microwave devices. In this paper, the author reports on the microstructure and magnetic properties of zinc ferrite thin films prepared by PLD.

\section{Experimental}

The bulk, $\mathrm{ZnFe}_{2} \mathrm{O}_{4}$ target material for ablation was prepared by using a ceramic technique starting from $\mathrm{Fe}_{2} \mathrm{O}_{3}$ and $\mathrm{ZnO}$ powders. The appropriate quantities of powders were carefully mixed together in a mortar and pestle and prefired at $800^{\circ} \mathrm{C}$ for $4 \mathrm{~h}$. After subsequent ball milling under isopropyl alcohol for $48 \mathrm{~h}$ the powders were pressed at $11 \mathrm{KN} / \mathrm{cm}^{2}$ into disk shapes $(25 \mathrm{~mm}$ diameter, $5 \mathrm{~mm}$ thickness). The specimens were sintered at $1200^{\circ} \mathrm{C}$

*Paper presented at 8 AGM of MRSI, BARC, Mumbai, 1997. for $4 \mathrm{~h}$ in a nitrogen atmosphere and then slowly cooled in the same atmosphere to room temperature.

The excimer laser (Lambda Physik model LEXtra 200) was operated at a frequency of $30 \mathrm{~Hz}$ with $\mathrm{XeCl}$ mixture, wavelength $308 \mathrm{~nm}$ and an energy of $225 \mathrm{~mJ}$ per pulse for the deposition. Films were deposited from the target onto $\mathrm{MgO}$ substrates heated to $600^{\circ} \mathrm{C}$ in an oxygen atmosphere of $100 \mathrm{mT}$ Trr. The laser beam focused onto $3 \mathrm{~mm}^{2}$ spot on the surface of the ablation target and was incident on the target surface at an angle of incidence $45^{\circ}$. The target material was rotated at about $12 \mathrm{rpm}$ and the substrate was mounted opposite the above target and at a distance of $35 \mathrm{~mm}$. The substrate was mounted on a stainless steel heater plate using silver paint. The laser itself was operated with the above parameters for $\sim 60 \mathrm{~min}$. After deposition, the thin films were cooled slowly to room temperature by turning the power to the substrate heater and maintaining the oxygen pressure in the vacuum chamber to 100 mTorr of oxygen.

Studies on crystal structure were performed by X-ray diffractometer (XRD). The surface texture, cross-section morphology and grain size were observed by JEOL-6400 scanning electron microscopy and atomic force microscopy. Magnetic measurements were performed in the temperature range $5-300 \mathrm{~K}$ and in fields of up to $5 \mathrm{~T}$ using a Quantum Design Squid magnetometer. Fields could be set to an accuracy of $+10^{-6} \mathrm{~T}$ and temperatures could be controlled to within $+10^{-2} \mathrm{~K}$.

\section{Results and discussion}

The XRD pattern of a typical thin film is shown in figure 1. As can be seen from the figure, all the prominent 


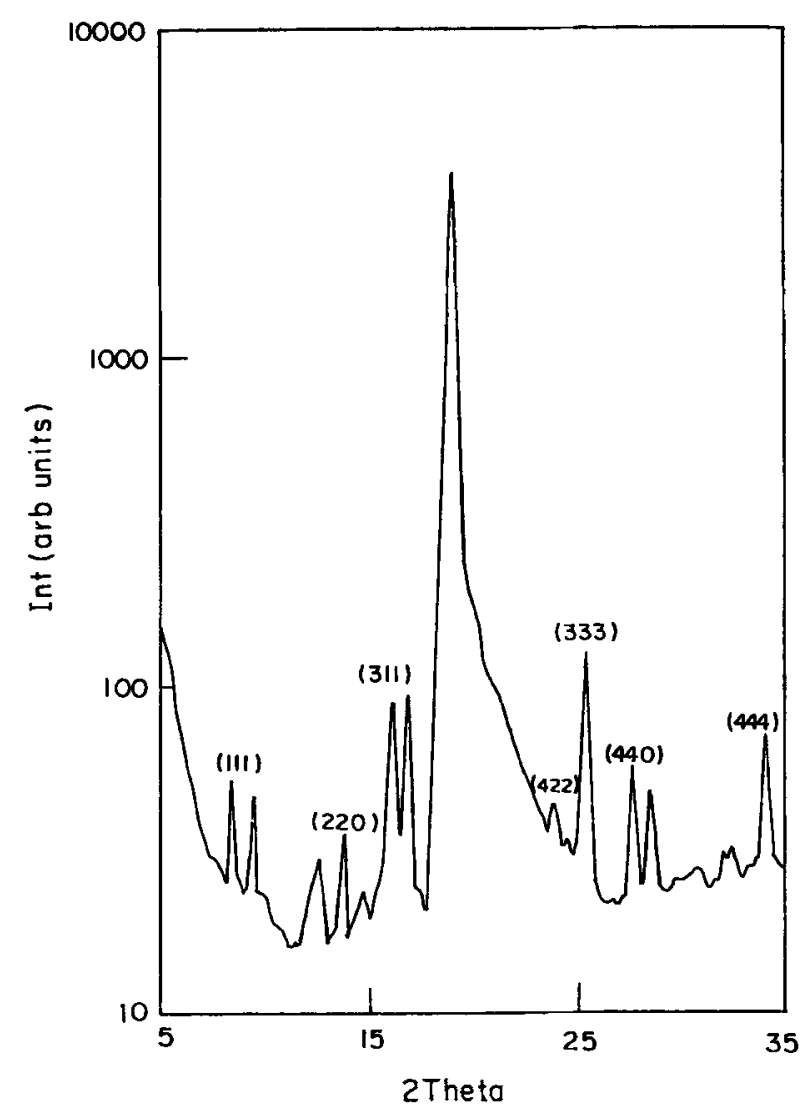

Figure 1. $\mathrm{X}$-ray diffractogram for zinc ferrite thin film grown on $\mathrm{MgO}$ substrate. lines corresponding to the bulk zinc ferrite were observed. All the intensity peaks were seen, showing the crystallization and the polycrystalline nature of the film. The calculated lattice constant of the zinc ferrite thin film was determined to be $0.8390 \mathrm{~nm}$ which is very close to bulk target material $(0.8430 \mathrm{~nm})$ and a decrease of $0.04 \mathrm{~nm}$ from the target material. This might be related to lattice mismatch between the zinc ferrite and $\mathrm{MgO}$ substrate which results in film strain.

Film surface morphology was examined using SEM. Figure 2 shows that the film surface is smooth, visibly the films were red in colour and almost specular with cloudy appearance. The droplet contamination, usually associated with PLD process was almost totally absent--this was due to the low laser fluence used for deposition. The overall surface texture was determined by crystallites with a grain size of $100 \mathrm{~nm}$. The chemical composition of the film was determined by energy dispersive X-ray spectroscopy. Film with composition $0.40 \mathrm{ZnO}, 0.60 \mathrm{Fe}_{2} \mathrm{O}_{3}$ was obtained. Thus, the film was rich in iron, but deficient in zinc. Atomic force microscopy is a powerful tool for studying grain morphology and surface topography. In this work AFM images were obtained using two modes: contact and tapping. In contact mode, the cantilever was always in contact with the surface of the sample while in tapping mode the cantilever oscillated close to its mechanical resonance frequency. Figure 3 a shows the surface topography of a film. The sample surface was smooth and consisted of hexagonal crystallites and several types of

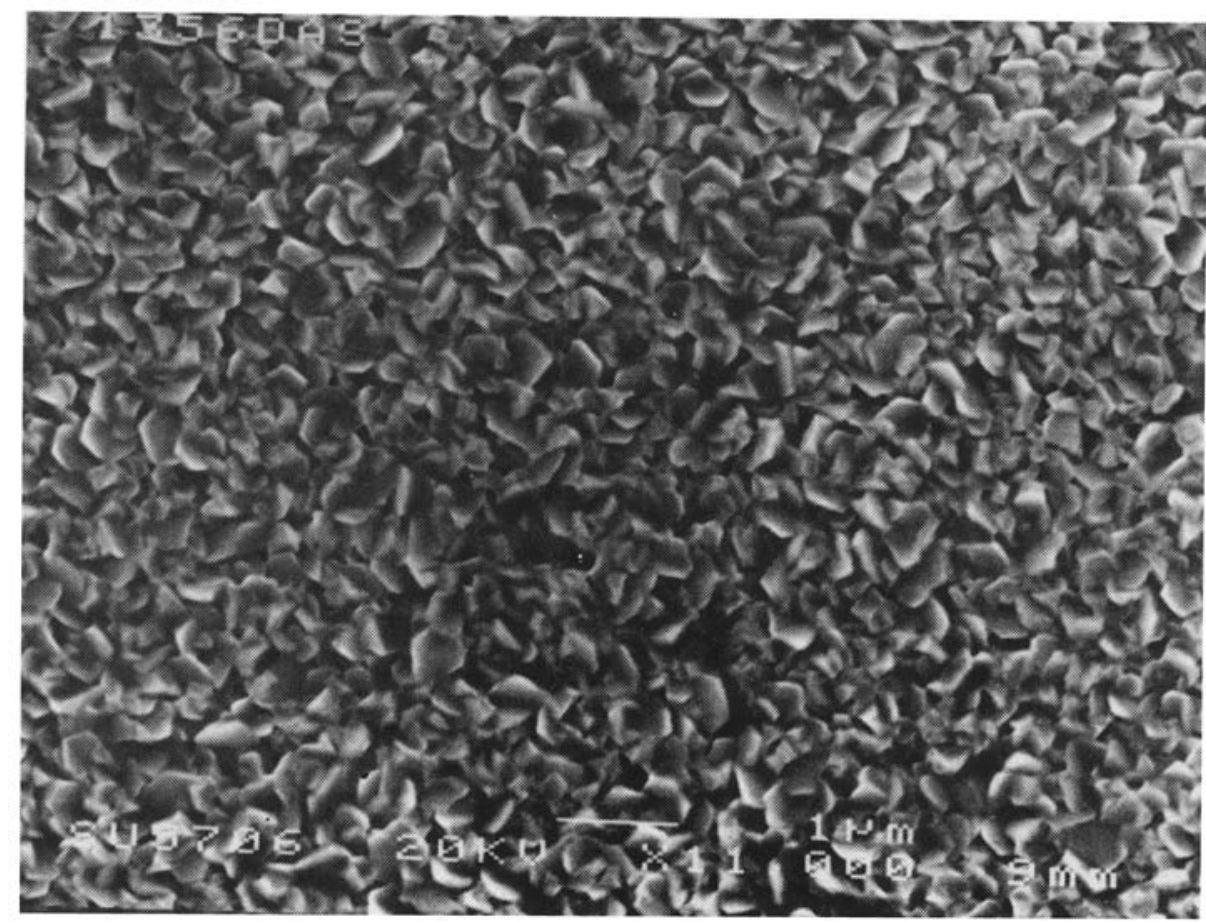

Figure 2. SEM micrograph of a zinc lerrite thin film. 


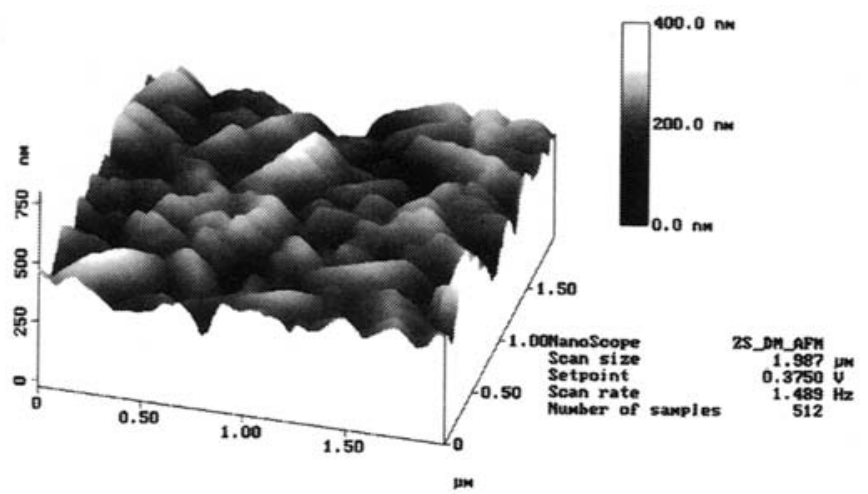

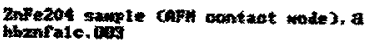

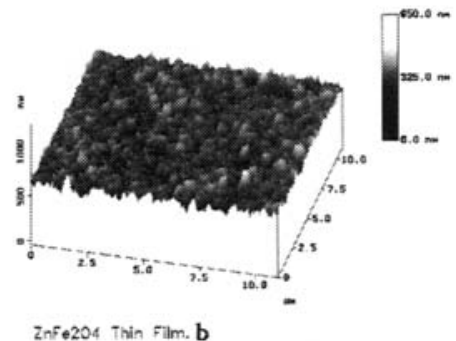

Figure 3. a. AFM image of zinc ferrite thin film at $2.0 \mu \mathrm{m}$ scan size and b. oblique view of a $10 \times 10 \mu \mathrm{m}^{2}$ surface of a $\mathrm{ZnFe}_{2} \mathrm{O}_{4}$ film obtained with an AFM. Microcrystals of about $150 \mathrm{~nm}$ width are well visible. outgrowths. These included particles with a diameter of $100-150 \mathrm{~nm}$, smaller spherical of irregular features and needle-shaped grains. It can be seen from figure $3 \mathrm{~b}$ which revealed the presence of numerous pyramidal hills.

Hysteresis loops were measured for the thin film in temperature range $5-300 \mathrm{~K}$. Figure 4 gives the temperature dependence of the remanence of a sample as determined from the full loops. An extrapolation of this curve to zero remanence gives a value of $75 \mathrm{~K} \pm 5 \mathrm{~K}$ for $T_{\mathrm{B}}$, the blocking temperature of the sample. This value is in very close agreement with a previous value quoted for zinc ferrite thin film produced by the rf sputtering method (Chen et al 1995). We have inferred a superparamagnetic behaviour for the zinc ferrite thin films from magnetization vs field data at a series of temperatures. The two criteria for such a behaviour are: (a) the magnetization above the blocking temperature must scale when plotted as a function of $H / T$, (b) $M$ vs $H$ data should show hysteresis below the blocking temperature but no hysteresis above the blocking temperature (Bean and Jacobs 1956). Superparamagnetism arise in materials consisting of single domain ferromagnetic or ferromagnetic particles when the thermal energy is large enough to cause the magnetization to undergo random fluctuations (Bean and Livingston 1959). Such a system is expected to behave like a Langevin paramagnet and the magnetization must scale as $H / T$. Figure 5 shows the variation of $M$ with $H / T$. It can be seen from the figure that the criterion for superparamagnetism is satisfied above the blocking temperature. Figures 6 and 7 show $M$ vs $H$ data at 5 and

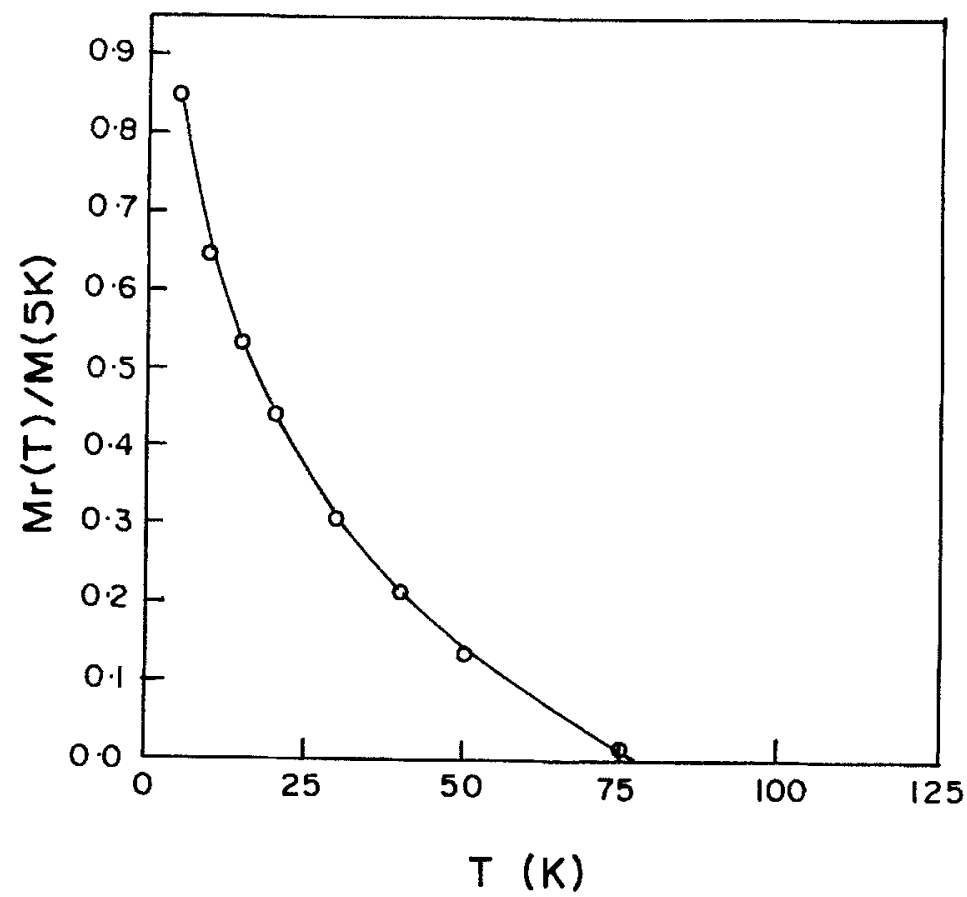

Figure 4. Temperature dependence of the remanence for a zinc ferrite thin film as derived from the corresponding hysteresis loops where a field of $5 \mathrm{~T}$ was applied at each temperature. The remanence is normalized to its value at $5 \mathrm{~K}$. 


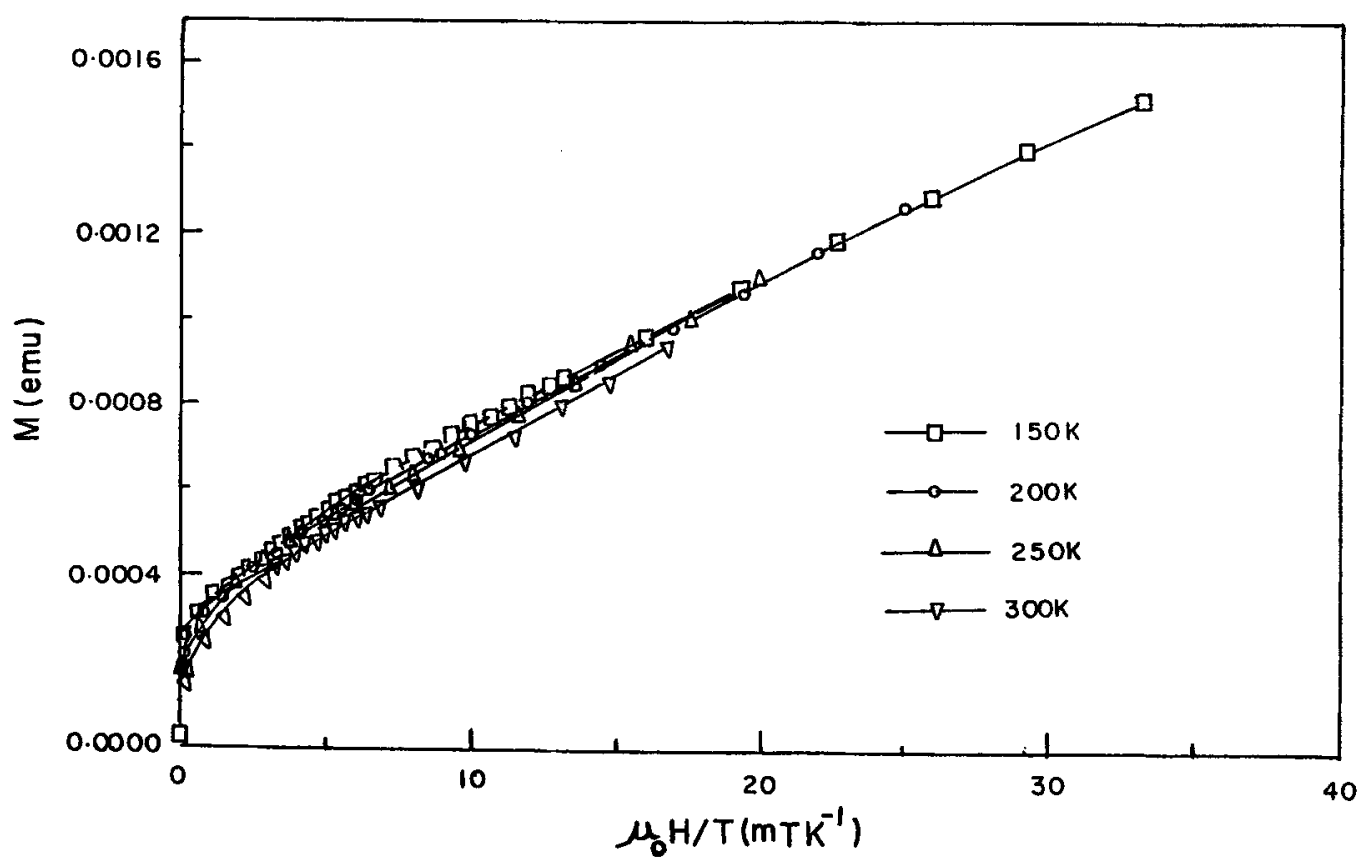

Figure 5. Magnetization of a zinc ferrite thin film plotted as a function of reduced field above blocking temperature.

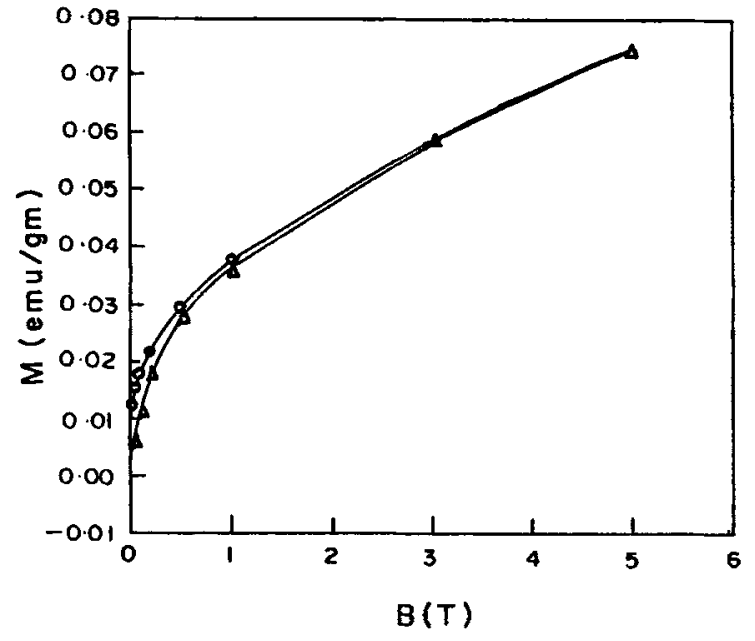

Figure 6. Magnetic field dependence of the magnetization for zinc ferrite thin film at $5 \mathrm{~K}$. The figure indicates hysteresis at $5 \mathrm{~K}$.

$300 \mathrm{~K}$. The data at $5 \mathrm{~K}$ show hysteresis, whereas the data at $300 \mathrm{~K}$ does not show any hysteresis. Thus, data in figures 6 and 7 lead us to the conclusion that zinc ferrite nanocrystals in the films are superparamagnetic. To the best of our knowledge, this is the first observation of superparamagnetism in pulsed laser ablated ferrite thin films.

\section{Acknowledgements}

The author is grateful to the Department of Science and Technology, New Delhi for the award of BOYSCAST fellowship. $\mathrm{He}$ is also grateful to $\mathrm{Dr} \mathrm{H} \mathrm{J}$ Blythe,

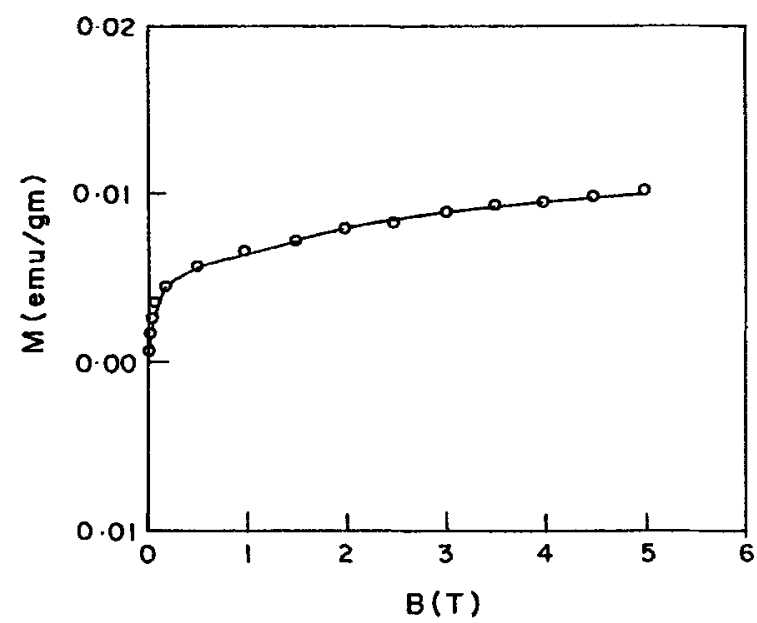

Figure 7. Magnetic field dependence of the magnetization for zinc ferrite thin film at $300 \mathrm{~K}$. The figure indicates no hysteresis at $300 \mathrm{~K}$.

Department of Physics, The University of Sheffield, for providing experimental facilities.

\section{References}

Bean C P and Jacobs I S 1956 J. Appl. Phys. 271448

Bean C P and Livingston J D 1959 Appl. Phys. 30120

Chen J, Srinivasan G, Hunter S, Suresh Babu V and Sheera M S 1995 J. Magn. Magn. Mater. 146291

Jackson T J and Palmer S B 1994 J. Phys. D: Appl. Phys. 27 1581

Sunita Gangopadhyay et al 1995 J. Magn. Magn. Mater. 147 L2

Welech R G, Jackson T J and Palmer S B 1995 IEEE Trans. Mag. 312752 